\title{
Uniform Persistence, Periodicity and Extinction in a Delayed Biological System with Stage Structure and Density-Dependent Juvenile Birth Rate
}

\author{
Limin Zhang1,2*, Chaofeng Zhang1 \\ ${ }^{1}$ School of Mathematics and Finance-Economics, Sichuan University of Arts and Science, Dazhou, China \\ ${ }^{2}$ College of Mathematics, Sichuan University, Chengdu, China \\ Email: "Imzhang2000@163.com
}

Received 20 May 2016; accepted 17 June 2016; published 20 June 2016

Copyright (C 2016 by authors and Scientific Research Publishing Inc.

This work is licensed under the Creative Commons Attribution International License (CC BY).

http://creativecommons.org/licenses/by/4.0/

(c) $\underset{\text { BY }}{ }$ Open Access

\begin{abstract}
A delayed biological system of predator-prey interaction with stage structure and density dependent juvenile birth rate is investigated. It is assumed that the prey population has two stages: immature and mature. The growth of the immature prey is density dependent and is a function of the density of adult prey. Such phenomenon has been reported for beetles, tribolium, copepods, scorpions, several fish species and even crows. The growth of the predator is affected by the time delay due to gestation. By some Lemmas and methods of delay differential equation, the conditions for the uniform persistence and extinction of the system are obtained. Numerical simulations illustrate the feasibility of the main results and demonstrate that the density dependent coefficient has influence on the system populations' densities though it has no effect on uniform persistence and extinction of the system.
\end{abstract}

\section{Keywords}

Uniform Persistence, Periodicity, Extinction, Density Dependence, Stage Structure

\section{Introduction}

In the natural world, there are many species whose individual members have a life history that takes them through two stages: juvenile and adult. Individuals in each stage are identical in biological characteristics, and

*Corresponding author.

How to cite this paper: Zhang, L.M. and Zhang, C.F. (2016) Uniform Persistence, Periodicity and Extinction in a Delayed Biological System with Stage Structure and Density-Dependent Juvenile Birth Rate. American Journal of Computational Mathematics, 6, 130-140. http://dx.doi.org/10.4236/ajcm.2016.62014 
some vital rates (rates of survival, development and recruitment) of individuals in a population almost always depend on stage structure [1]-[3]. Thus, we need to consider stage structure in population problems accordingly. In recent years, some authors ([1]-[18]) studied the stage-structured predator-prey systems. The authors of [2]-[11] have studied the stability or Hopf bifurcation of these type systems. Since environmental and biological parameters (such as the seasonal effects of weather, food supplies, mating habits, hunting or harvesting season, etc.) fluctuate naturally over time, the authors of [12]-[18] have explored a class of nonautonomous biological systems with stage structure. Recently, Yang et al. considered the following predator-prey system with stage structure for prey [18]:

$$
\begin{aligned}
& \dot{x}_{1}(t)=a(t) x_{2}(t)-b(t) x_{1}(t)-d(t) x_{1}^{2}(t)-\frac{p(t) x_{1}(t)}{k(t)+x_{1}^{2}(t)} y(t), \\
& \dot{x}_{2}(t)=c(t) x_{1}(t)-f(t) x_{2}^{2}(t), \\
& \dot{y}(t)=y(t)\left(-g(t)+\frac{h(t) x_{1}(t)}{k(t)+x_{1}^{2}(t)}-q(t) y(t)\right) .
\end{aligned}
$$

All the coefficients in system (1.1) are continuous positive $\omega$ periodic functions. Sufficient and necessary conditions are obtained for the permanence of the system.

Sometimes, the past state as well as current conditions can influence biological dynamics and such interactions have motivated the introduction of time delay in stage-structured predator-prey systems [2]-[5] [8]-[13]. Time delay due to gestation is the time interval between the moments when an individual prey is killed and when the corresponding biomass is added to the predator population. That is to say, the reproduction of predator after predating the prey is not instantaneous but will be mediated by some discrete time lag required for gestation of predator. The authors of [8]-[10] have studied a class of stage-structured predator-prey models with time delay due to gestation of predator.

In some stage-structured populations, the intraspecific and interspecific competitions occur within each stage structure. And each stage-structured density affects not only its population but also other stage-structured populations. In two-stage single-species population, Abrams and Quince have demonstrated that adult population competition makes a low birth rate of juvenile population [19]. Adult population has to compete for resources to reproduce when population size or density is larger. Correspondingly, juvenile population birth rate is a function of adults' density and remains bounded when adults' size is large due to limited resources [20]. This densitydependent regulator has been found in beetles, tribolium, copepods, scorpions, several fish species and even crows by Polis [21].

Motivated by the above facts and based on the recent work of Yang et al. [18], we consider the following stage-structured predator-prey model:

$$
\begin{aligned}
& \dot{x}_{1}(t)=a(t)\left(1-\beta(t) x_{2}(t)\right) x_{2}(t)-b(t) x_{1}(t)-d_{1}(t) x_{1}(t)^{2}-\frac{c_{1}(t) x_{1}(t) y(t)}{m(t)+x_{1}^{2}(t)}, \\
& \dot{x}_{2}(t)=b(t) x_{1}(t)-d_{2}(t) x_{2}^{2}(t), \\
& \dot{y}(t)=y(t)\left(-d_{3}(t)+\frac{c_{2}(t) x_{1}(t-\tau)}{m(t)+x_{1}^{2}(t-\tau)}-q(t) y(t)\right),
\end{aligned}
$$

where $x_{1}(t), x_{2}(t)$ and $y(t)$ represent the density of immature prey, mature prey and predator species, respectively. The coefficients in system (1.2) are all continuous positive $T$ periodic functions in which $a(t)$ represents the maximum per capita birth rate into the immature prey, $b(t)$ is the recruitment rate of immature prey becoming mature prey, $d_{1}(t)$ is the death rate of the immature prey population, and $d_{2}(t)$ is death rate of the mature prey population. The parameter $\beta(t)$ is the proportional rate of decrease in per capita births with increased mature prey density and takes a value between 0 and 1 [19], which can be considered as density dependent coefficient. The function $c_{1}(t) x_{1}(t) /\left(m(t)+x_{1}^{2}(t)\right)$ represents the Holling type-IV functional response of the predator to the immature prey and $c_{2}(t) / c_{1}(t)$ is the conversion rate of nutrients into the reproduction of the predator. The parameter $\tau$ is the delay due to gestation, that is, only the mature adult predator can contribute to the production of predator biomass. The parameters $d_{3}(t)$ and $q(t)$ denote the death rate 
and the overcrowding rate of the predator population, respectively.

The initial conditions for system (1.2) take the form of

$$
x_{i}(\theta)=\phi_{i}(\theta), y(\theta)=\psi(\theta), \phi_{i}(0)>0, \psi(0)>0, i=1,2, \theta \in[-\tau, 0],
$$

where $\left(\phi_{1}(\theta), \phi_{2}(\theta), \psi(\theta)\right) \in C\left([-\tau, 0], R_{+0}^{3}\right)$, the Banach space of continuous functions mapping the interval $[-\tau, 0]$ into $R_{+0}^{3}$, where we define $R_{+0}^{3}=\left\{\left(x_{1}, x_{2}, x_{3}\right) \mid x_{i} \geq 0, i=1,2,3\right\}$ and the interior of $R_{+0}^{3}$ as $R_{+}^{3}=\left\{\left(x_{1}, x_{2}, x_{3}\right) \mid x_{i}>0, i=1,2,3\right\}$.

At the same time, we adopt the following notations through this paper:

$$
A_{T}(g)=\frac{1}{T} \int_{0}^{T} g(t) \mathrm{d} t, g^{M}=\sup _{t \in[0, T]} g(t), g^{l}=\inf _{t \in[0, T]} g(t) .
$$

where $g(t)$ is a continuous $T$-periodic function.

The remainder of this paper is organized as follows. In Section 2, we introduce some lemmas and then explore the uniformly persistence and periodicity of system (1.2). In Section 3, we investigate the extinction of the predator population in system (1.2). In Section 4, numerical simulations are presented to illustrate the feasibility of our main results. Conclusion is given in Section 5 .

\section{Uniform Persistence and Periodicity}

In this section, we analyze the uniform persistence and periodicity of system (1.2) with initial conditions (1.3). First, we introduce the following definition and lemmas, which are useful for obtaining our results.

Definition 2.1. The system $\dot{x}(t)=F(t, x), x \in R^{n}$ is said to be uniformly persistent if there are constants $M \geq m>0$ such that every positive solution of this system satisfies $m \leq \liminf _{t \rightarrow \infty} x_{i}(t) \leq \limsup _{t \rightarrow \infty} x_{i}(t) \leq M$.

Definition 2.2. The system $\dot{x}(t)=F(t, x), \quad x \in R^{n}$ is said to be weakly uniformly persistent if there are constants $\eta>0$ such that every positive solution of this system satisfies $\limsup x_{i}(t) \geq \eta$.

Lemma 2.1. (See [22]). If $a(t)$ and $b(t)$ are all continuous $T$ periodic functions for all $t \in R$, and $A_{T}(a(t))>0, \quad b(t)>0$ then the system

$$
\dot{x}(t)=x(t)(a(t)-b(t) x(t)),
$$

has a unique positive $T$ periodic solution which is globally asymptotically stable.

Lemma 2.2. (See [23]). If $a(t), b(t), d_{1}(t)$ and $d_{2}(t)$ are all positive and continuous $T$ periodic functions for all $t \in R$, then the system

$$
\begin{aligned}
& \dot{x}_{1}(t)=a(t) x_{2}(t)-b(t) x_{1}(t)-d_{1}(t) x_{1}^{2}(t), \\
& \dot{x}_{2}(t)=b(t) x_{1}(t)-d_{2}(t) x_{2}^{2}(t),
\end{aligned}
$$

has a positive T periodic solution $\left(x_{1}^{*}(t), x_{2}^{*}(t)\right)$ which is globally asymptotically stable with respect to $R_{+0}^{2}=\left\{\left(u_{1}, u_{2}\right) \mid u_{1}>0, u_{2}>0\right\}$.

Theorem 2.1. System (1.2) is uniformly persistent and has at least one positive T periodic solution provided that

$$
A_{T}\left(-d_{3}(t)+\frac{c_{2}(t) x_{1}^{*}(t-\tau)}{m(t)+\left(x_{1}^{*}(t-\tau)\right)^{2}}\right)>0,
$$

where $\left(x_{1}^{*}(t), x_{2}^{*}(t)\right)$ is the unique positive periodic solution of system (2.2) given by Lemma 2.2.

We need the following propositions to prove Theorem 2.1.

Proposition 2.1. There exists a positive constant $M_{x}$ and $M_{y}$ such that

$$
\lim _{t \rightarrow+\infty} \sup x_{i}(t) \leq M_{x}(i=1,2), \lim _{t \rightarrow+\infty} \sup y(t) \leq M_{y} .
$$

Proof. Obviously, $R_{+}^{3}$ is a positively invariant set of system (1.2). Given any solution $\left(x_{1}(t), x_{2}(t), y(t)\right)$ of system (1.2) with initial conditions (1.3), we have 


$$
\begin{gathered}
\dot{x}_{1}(t) \leq a(t) x_{2}(t)-b(t) x_{1}(t)-d_{1}(t) x_{1}^{2}(t), \\
\dot{x}_{2}(t)=b(t) x_{1}(t)-d_{2}(t) x_{2}^{2}(t) .
\end{gathered}
$$

Consider the following auxiliary system:

$$
\begin{aligned}
& \dot{u}_{1}(t)=a(t) u_{2}(t)-b(t) u_{1}(t)-d_{1}(t) u_{1}^{2}(t), \\
& \dot{u}_{2}(t)=b(t) u_{1}(t)-d_{2}(t) u_{2}^{2}(t) .
\end{aligned}
$$

By Lemma 2.2, system (2.4) has a unique globally attractive positive $T$ periodic solution $\left(x_{1}^{*}(t), x_{2}^{*}(t)\right)$. Let $\left(u_{1}(t), u_{2}(t)\right)$ be the solution of system (2.4) with $\left(u_{1}(0), u_{2}(0)\right)=\left(x_{1}(0), x_{2}(0)\right)$. By the vector comparison theorem [24], we have

$$
x_{i}(t) \leq u_{i}(t)(i=1,2), t \geq 0 .
$$

From the global attractivity of $\left(x_{1}^{*}(t), x_{2}^{*}(t)\right)$, for any positive constant $\varepsilon(0<\varepsilon<1)$, there exists a $T_{1}>0$, such that for all $t \geq T_{1}$

$$
\left|u_{i}(t)-x_{i}^{*}(t)\right|<\varepsilon, \quad i=1,2
$$

By applying (2.5) and (2.6), we obtain $x_{i}(t) \leq x_{i}^{*}(t)+\varepsilon, \quad i=1,2, \quad t \geq T_{1}$. Let $M_{x}=\max _{0 \leq t \leq T}\left\{x_{i}^{*}(t)+\varepsilon, i=1,2\right\}$, we have $\lim _{t \rightarrow+\infty} \sup x_{i}(t) \leq M_{x}(i=1,2)$. In addition, from the third equation of (1.2) we have

$$
\dot{y}(t) \leq y(t)\left(\frac{c_{2}(t) M_{x}}{m(t)}-q(t) y(t)\right),
$$

for all $t \geq T_{1}$. Consider the following auxiliary equation:

$$
\dot{v}(t)=v(t)\left(\frac{c_{2}(t) M_{x}}{m(t)}-q(t) v(t)\right)
$$

By Lemma (2.1), we obtain that system (2.7) has a unique positive $T$ periodic solution $y^{*}(t)>0$ which is globally asymptotically stable. Similarly to the above analysis, for the above $\varepsilon$, there exists a $T_{2}>T_{1}$, such that

$$
y(t)<y^{*}(t)+\varepsilon, t \geq T_{2} .
$$

Set $M_{y}=\max _{0 \leq t \leq T}\left\{y^{*}(t)+\varepsilon\right\}$, then $\lim _{t \rightarrow+\infty} \sup y(t) \leq M_{y}$.

Proposition 2.2. There exists a positive constant $\eta_{x}<M_{x}$, such that

$$
\lim _{t \rightarrow+\infty} \inf x_{i}(t) \geq \eta_{x}, \quad i=1,2 \text {. }
$$

Proof. By Proposition 2.1, there exists a positive $T_{2}>0$ such that $0<y(t)<M_{y}, t \geq T_{2}$. Hence, from the first and second equations of system (1.2), we obtain

$$
\begin{gathered}
\dot{x}_{1}(t) \geq a(t)\left(1-\beta(t) M_{x}\right) x_{2}(t)-\left(b(t)+\frac{c_{1}(t) M_{y}}{m(t)}\right) x_{1}(t)-d_{1}(t) x_{1}^{2}(t) \\
\dot{x}_{2}(t)=b(t) x_{1}(t)-d_{2}(t) x_{2}^{2}(t),
\end{gathered}
$$

for $t \geq T_{2}$. By Lemma 2.2, the following auxiliary system

$$
\begin{aligned}
& \dot{u}_{1}(t)=a(t)\left(1-\beta(t) M_{x}\right) u_{2}(t)-\left(b(t)+\frac{c_{1}(t) M_{y}}{m(t)}\right) u_{1}(t)-d_{1}(t) u_{1}^{2}(t) \\
& \dot{u}_{2}(t)=b(t) u_{1}(t)-d_{2}(t) u_{2}^{2}(t),
\end{aligned}
$$

has a unique global attractive positive $T$ periodic solution $\left(\tilde{x}_{1}^{*}(t), \tilde{x}_{2}^{*}(t)\right)$. Let $\left(u_{1}(t), u_{2}(t)\right)$ be the solution of system (2.9) with $\left(u_{1}\left(T_{2}\right), u_{2}\left(T_{2}\right)\right)=\left(x_{1}\left(T_{2}\right), x_{2}\left(T_{2}\right)\right)$, by the vector comparison theorem, we obtain 


$$
x_{i}(t) \geq u_{i}(t)(i=1,2), t \geq T_{2} .
$$

Moreover, from the global attractivity of $\left(\tilde{x}_{1}^{*}(t), \tilde{x}_{2}^{*}(t)\right)$, there exists a $T_{3}>T_{2}$, such that

$$
\left|u_{i}(t)-\tilde{x}_{i}^{*}(t)\right|<\frac{\tilde{x}_{i}^{*}(t)}{2}, t \geq T_{3} .
$$

Combined (2.10) with (2.11), we have

$$
x_{i}(t)>\min _{t \in[0, T]}\left\{\frac{\tilde{x}_{i}^{*}(t)}{2}, i=1,2\right\}=\eta_{x}, t \geq T_{3} .
$$

Therefore, $\lim _{t \rightarrow+\infty} \inf x_{i}(t) \geq \eta_{x}, \quad i=1,2$.

Proposition 2.3. Suppose that (2.3) holds, then there exists a positive constant $\eta_{y}$, such that any solution $\left(x_{1}(t), x_{2}(t), y(t)\right)$ of system (1.2) with initial conditions (1.3) satisfies

$$
\lim _{t \rightarrow+\infty} \sup y(t) \geq \eta_{y} \text {. }
$$

Proof. By assumption (2.3), we can choose arbitrarily small constant $\varepsilon_{0}>0$ (without loss generality, we assume that $0<\varepsilon_{0}<\frac{1}{2} \min _{t \in[0, T]}\left\{x_{1}^{*}(t), x_{2}^{*}(t)\right\}$, where $\left(x_{1}^{*}(t), x_{2}^{*}(t)\right)$ is the unique positive periodic solution of system (2.2)), such that

$$
A_{T}\left(\Phi_{\varepsilon_{0}}(t)\right)>0,
$$

where $\Phi_{\varepsilon_{0}}=-d_{3}(t)+\frac{c_{2}(t)\left(x_{1}^{*}(t-\tau)-\varepsilon_{0}\right)}{m(t)+\left(\left(x_{1}^{*}(t-\tau)\right)^{2}-\varepsilon_{0}\right)}-q(t) \varepsilon_{0}$. Consider the following system with a parameter $\delta$,

$$
\begin{aligned}
& \dot{x}_{1}(t)=a(t)\left(1-\beta(t) M_{x}\right) x_{2}(t)-\left(b(t)+\frac{2 c_{1}(t) \delta}{m(t)}\right) x_{1}(t)-d_{1}(t) x_{1}^{2}, \\
& \dot{x}_{2}(t)=b(t) x_{1}(t)-d_{2}(t) x_{2}^{2}(t) .
\end{aligned}
$$

By Lemma 2.2, system (2.14) has a unique positive $T$ periodic solution $\left(x_{1 \delta}^{*}(t), x_{2 \delta}^{*}(t)\right)$, which is globally attractive. Let $\left(\bar{x}_{1 \delta}(t), \bar{x}_{2 \delta}(t)\right)$ be the solution (2.14) with initial condition $\left(\bar{x}_{1 \delta}(0), \bar{x}_{2 \delta}(0)\right)=\left(x_{1}^{*}(0), x_{2}^{*}(0)\right)$. Then, for the above $\varepsilon_{0}$, there exists a sufficiently large $T_{4}>T_{3}$ such that $\left|\bar{x}_{i \delta}(t)-x_{i \delta}^{*}(t)\right|<\frac{\varepsilon_{0}}{4}$, for $i=1,2$, $t>T_{4}$. Using the continuity of the solution in the parameter, we have $\bar{x}_{i \delta}(t) \rightarrow x_{i}^{*}(t)$ uniformly in $\left[T_{4}, T_{4}+T\right]$ as $\delta \rightarrow 0$. Hence, there exists a $\delta_{0}=\delta_{0}\left(\varepsilon_{0}\right)>0$ such that $\left|\bar{x}_{i \delta}(t)-x_{i}^{*}(t)\right|<\frac{\varepsilon_{0}}{4}$, for $i=1,2$, $t \in\left[T_{4}, T_{4}+T\right], \quad 0<\delta<\delta_{0}$. So, we get $\left|x_{i \delta}^{*}(t)-x_{i}^{*}(t)\right| \leq\left|\bar{x}_{i \delta}(t)-x_{i \delta}^{*}(t)\right|+\left|\bar{x}_{i \delta}(t)-x_{i}^{*}(t)\right|<\frac{\varepsilon_{0}}{2}$. Choosing a constant $\delta_{1}\left(0<\delta_{1}<\delta_{0}, 2 \delta_{1}<\varepsilon_{0}\right)$, we obtain

$$
x_{i \delta_{1}}^{*}(t) \geq x_{i}^{*}(t)-\frac{\varepsilon_{0}}{2}, i=1,2, t \geq 0 .
$$

Suppose that the conclusion (2.12) is not true, then there exists $\psi \in C_{+}$such that

$$
\lim _{t \rightarrow+\infty} \sup y(t)<\delta_{1} \text {, }
$$

where $\left(x_{1}(t, \psi), x_{2}(t, \psi), y(t, \psi)\right)$ is the solution of system (1.2) with initial condition $\left(x_{1}(\theta), x_{2}(\theta), y(\theta)\right)=\psi(\theta), \theta \in[-\tau, 0]$. So, there exists $T_{5}>T_{4}$ such that

$$
y(t)<2 \delta_{1}<\varepsilon_{0},
$$


for $t \geq T_{5}$. Then, from the first and second equation of system (1.2), we have

$$
\begin{aligned}
& \dot{x}_{1}(t, \phi) \geq a(t)\left(1-\beta(t) M_{x}\right) x_{2}(t, \phi)-\left(b(t)+\frac{2 c_{1}(t) \sigma_{1}}{m(t)}\right) x_{1}(t, \phi)-d_{1}(t) x_{1}^{2}(t, \phi), \\
& \dot{x}_{2}(t, \phi)=b(t) x_{1}(t, \phi)-d_{2}(t) x_{2}^{2}(t, \phi),
\end{aligned}
$$

for all $t \geq T_{6} \geq T_{5}+\tau_{1}$. Let $\left(u_{1}(t), u_{2}(t)\right)$ be the solution of system (2.14) with $\delta=\delta_{1}$ and $\left(u_{1}\left(T_{6}\right), u_{2}\left(T_{6}\right)\right)=\left(x_{1}\left(T_{6}, \psi\right), x_{2}\left(T_{6}, \psi\right)\right)$, then we have

$$
x_{i}(t, \psi) \geq u_{i}(t), i=1,2, t \geq T_{6} .
$$

By the global asymptotic stability of $\left(x_{1 \delta_{1}}^{*}(t), x_{2 \delta_{1}}^{*}(t)\right)$, for the given $\varepsilon=\varepsilon_{0} / 2$, there exists $T_{7} \geq T_{6}$, such that

$$
\left|u_{i}(t)-x_{i \delta_{1}}^{*}(t)\right|<\frac{\varepsilon_{0}}{2}, i=1,2, t \geq T_{7} .
$$

So, $x_{i}(t, \psi) \geq u_{i}(t)>x_{i \delta_{1}}^{*}(t)-\frac{\varepsilon_{0}}{2}, \quad i=1,2, t \geq T_{7}$. By using (2.15), we obtain

$$
x_{i}(t, \psi) \geq x_{i}^{*}(t)-\varepsilon_{0}, i=1,2, t \geq T_{7} .
$$

Therefore, by using (2.17) and (2.19), for $t \geq T_{7}+\tau$ it follows:

$$
\dot{y}(t, \phi) \geq y(t, \phi)\left(-d_{3}(t)+\frac{c_{2}(t)\left(x_{1}^{*}(t-\tau)-\varepsilon_{0}\right)}{m(t)+\left(\left(x_{1}^{*}(t-\tau)\right)^{2}-\varepsilon_{0}\right)}-q(t) \varepsilon_{0}\right)=y(t, \phi) \Phi_{\varepsilon_{0}}
$$

Integrating (2.20) from $T_{7}+\tau$ to $t$ yields

$$
y(t, \psi) \geq y\left(T_{7}+\tau, \psi\right) \exp \int_{T_{7}+\tau_{2}}^{t} \phi_{\varepsilon_{0}}(t) \mathrm{d} t .
$$

Thus, from (2.13) we know that $y(t, \psi) \rightarrow+\infty$ as $t \rightarrow+\infty$, which is a contradiction. The proof is complete.

Proof of Theorem 2.1. By Propositions2.2 and 2.3, system (1.2) is uniform weakly uniformly persistent. From Propositions 2.1 and Theorem 1.3.3 in [25], system (1.2) is uniformly persistent. Using result given by Xu, Chaplain and Davidson in [26] or Wang and Zhu in [27], we obtain system (1.2) has at least one positive $T$ periodic solution. This completes the proof of Theorem 2.1.

\section{Extinction}

In this section, we investigate the extinction of the predator population in system (1.2) with initial conditions (1.3) under some condition.

Theorem 3.1. In system (1.2), suppose that

$$
A_{T}\left(-d_{3}(t)+\frac{c_{2}(t) x_{1}^{*}(t-\tau)}{m(t)+\left(x_{1}^{*}(t-\tau)\right)^{2}}\right) \leq 0,
$$

where $\left(x_{1}^{*}(t), x_{2}^{*}(t)\right)$ is the unique positive periodic solution of system (2.2) given by Lemma 2.2, then $\lim _{t \rightarrow+\infty} y(t)=0$.

Proof. According to (3.1), for every given positive constant $\varepsilon \quad(0<\varepsilon<1)$, there exists $\varepsilon_{1}>0 \quad\left(0<\varepsilon_{1}<\varepsilon\right)$ and $\varepsilon_{0}>0$ such that

$$
A_{T}\left(-d_{3}(t)+\frac{c_{2}(t)\left(x_{1}^{*}(t-\tau)+\varepsilon_{1}\right)}{m(t)+\left(x_{1}^{*}(t-\tau)+\varepsilon_{1}\right)^{2}}-q(t) \varepsilon\right) \leq-\frac{\varepsilon}{2} A_{T}(q(t))<-\varepsilon_{0} .
$$


From the first and second equations of system (1.2), we have

$$
\begin{gathered}
\dot{x}_{1}(t) \leq a(t) x_{2}(t)-b(t) x_{1}(t)-d_{1}(t) x_{1}^{2}(t), \\
\dot{x}_{2}(t)=b(t) x_{1}(t)-d_{2}(t) x_{2}^{2}(t),
\end{gathered}
$$

Hence, for the above $\varepsilon_{1}$ there exists a $\Gamma^{(1)}>0$, such that

$$
x_{i}(t)<x_{i}^{*}(t)+\varepsilon_{1}, \quad i=1,2, t \geq \Gamma^{(1)} .
$$

It follows from (3.2) and (3.3), that for $t \geq \Gamma^{(1)}+\tau$,

$$
A_{T}\left(-d_{3}(t)+\frac{c_{2}(t) x_{1}(t-\tau)}{m(t)+x_{1}(t-\tau)^{2}}-q(t) \varepsilon\right)<-\varepsilon_{0}
$$

First, we show that exists a $\Gamma^{(2)}>\Gamma^{(1)}+\tau$, such that $y\left(\Gamma^{(2)}\right)<\varepsilon$. Otherwise, by (3.4), we have

$$
\begin{aligned}
\varepsilon & \leq y(t) \leq y\left(\Gamma^{(1)}+\tau\right) \exp \left\{\left(\int_{\Gamma^{(1)}+\tau_{2}}^{t}-d_{3}(t)+\frac{c_{2}(t) x_{1}(t-\tau)}{m(t)+x_{1}(t-\tau)^{2}}-q(t) \varepsilon\right) \mathrm{d} s\right\} \\
& \leq y\left(\Gamma^{(1)}+\tau\right) \exp \left\{-\varepsilon_{0}\left(t-\left(\Gamma^{(1)}+\tau\right)\right)\right\} \rightarrow 0
\end{aligned}
$$

as $t \rightarrow+\infty$.

That is to say $\varepsilon \leq 0$. This is a contradiction. Second, we show that

$$
y(t) \leq \varepsilon \exp \{M(\varepsilon) T\}, \text { for } t \geq \Gamma^{(2)},
$$

where $M(\varepsilon)=\max _{0 \leq t \leq T}\left(-d_{3}(t)+\frac{c_{2}(t) x_{1}(t-\tau)}{m(t)+x_{1}(t-\tau)^{2}}-q(t) \varepsilon\right)$ is bounded for $\varepsilon \in[0,1]$. Otherwise, there exists a $\Gamma^{(3)}>\Gamma^{(2)}$, such that $y\left(\Gamma^{(3)}\right)>\varepsilon \exp \{M(\varepsilon) T\}$. By the continuity of $y(t)$, there must exist a $\Gamma^{(4)} \in\left(\Gamma^{(2)}, \Gamma^{(3)}\right)$ such that $y\left(\Gamma^{(4)}\right)=\varepsilon$ and $y(t)>\varepsilon$ for $t \in\left(\Gamma^{(4)}, \Gamma^{(3)}\right]$. Let $P_{1}$ be the nonnegative integer such that $\Gamma^{(3)} \in\left(\Gamma^{(4)}+P_{1} T, \Gamma^{(4)}+\left(P_{1}+1\right) T\right)$. According to (3.3), we have

$$
\begin{aligned}
\varepsilon \exp \{M(\varepsilon) T\} & <y\left(\Gamma^{(3)}\right)<y\left(\Gamma^{(4)}\right) \exp \left\{\left(\int_{\Gamma^{(4)}}^{\Gamma^{(3)}}\left(-d_{3}(t)+\frac{c_{2}(t) x_{1}(t-\tau)}{m(t)+x_{1}(t-\tau)^{2}}-q(t) \varepsilon\right) \mathrm{d} t\right\}\right. \\
& <\varepsilon \exp \left\{\left(\int_{\Gamma^{(4)}+P_{1} T}^{\Gamma^{(3)}}\left(-d_{3}(t)+\frac{c_{2}(t) x_{1}(t-\tau)}{m(t)+x_{1}(t-\tau)^{2}}-q(t) \varepsilon\right) \mathrm{d} t\right\}<\varepsilon \exp \{M(\varepsilon) T\},\right.
\end{aligned}
$$

which is a contradiction. This shows that (3.5) holds. By the arbitrariness of $\varepsilon$, it follows immediately that $y(t) \rightarrow 0$ as $t \rightarrow 0$. This completes the proof of Theorem 3.1.

From Theorem 2.1 and 3.1, we obtain that the density dependent coefficient $\beta(t)$ has no influence on permanence and extinction of system 1.2. But, from the following simulation, we can know the density dependent coefficient has effect on the populations' densities of system (1.2).

\section{Examples}

In this section, we provide some examples to illustrate the feasibility of our main results in Theorems 2.1 and 3.1 and then explore the effect of density dependent coefficient $\beta(t)$.

Example 4.1. Let 


$$
\begin{aligned}
& a(t)=3, b(t)=2, d_{1}(t)=\exp (-0.6), c_{1}(t)=4, m_{1}(t)=5, \\
& d_{2}(t)=\exp (-0.6), d_{3}(t)=0.3+0.3 \sin t, \quad c_{2}(t)=2, q(t)=0.05, \tau=0.6 .
\end{aligned}
$$

In this case, system (2.2) given by Lemma 2.2 has a unique positive periodic solution $\left(x_{1}^{*}(t), x_{2}^{*}(t)\right)=(2.70,3.14)$. Furthermore, by a simple calculation, we have

$$
A_{T}\left(-d_{3}(t)+\frac{C_{2}(t) x_{1}^{*}(t-\tau)}{m(t)+\left(x_{1}^{*}(t-\tau)\right)^{2}}\right)=0.175>0 \text {. }
$$

Let $\beta(t)=0.01$, according to Theorem 2.1, system (1.2) with the above coefficients is uniformly persistent and admits at least one positive $2 \pi$-periodic solution. Figure 1 shows the dynamic behavior of system (1.2).

Let $\beta=0.25$, according to Theorem 2.1, system (1.2) with the above coefficients is uniformly persistent and admits at least one positive $2 \pi$-periodic solution. Figure 2 shows the dynamic behavior of system (1.2).

From Theorem 2.1, we know that the density dependent coefficient $\beta(t)$ has no influence on the uniform persistence of system (1.2). However, from Figure 1 and Figure 2, we can see that the density dependent coefficient $\beta(t)$ affects the populations' densities of system (1.2). Figure 1 demonstrates that the system have high densities with the low density dependent coefficient; whereas Figure 2 shows the system have low densities with the high density dependent coefficient.

Example 4.2. Let

$$
\begin{aligned}
& a(t)=3, \quad \beta=0.25, \quad b(t)=2, d_{1}(t)=\exp (-0.6), \quad c_{1}(t)=4, m_{1}(t)=5 \\
& d_{2}(t)=\exp (-0.6), d_{3}(t)=0.75+0.3 \sin t, \quad c_{2}(t)=2, q(t)=0.07, \tau=0.6
\end{aligned}
$$

In this case, by a simple calculation, we obtain $A_{T}\left(-d_{3}(t)+\frac{c_{2}(t) x_{1}^{*}(t-\tau)}{m(t)+\left(x_{1}^{*}(t-\tau)\right)^{2}}\right)=-0.582<0$. According to Theorem 3.1, system (1.2) is impermanent and the predator population experiences extinction. The numerical simulation shown in Figure 3 also confirms this result.

\section{Conclusion}

In this paper, we propose a stage-structured predator-prey system with time delay and density-dependent juve-

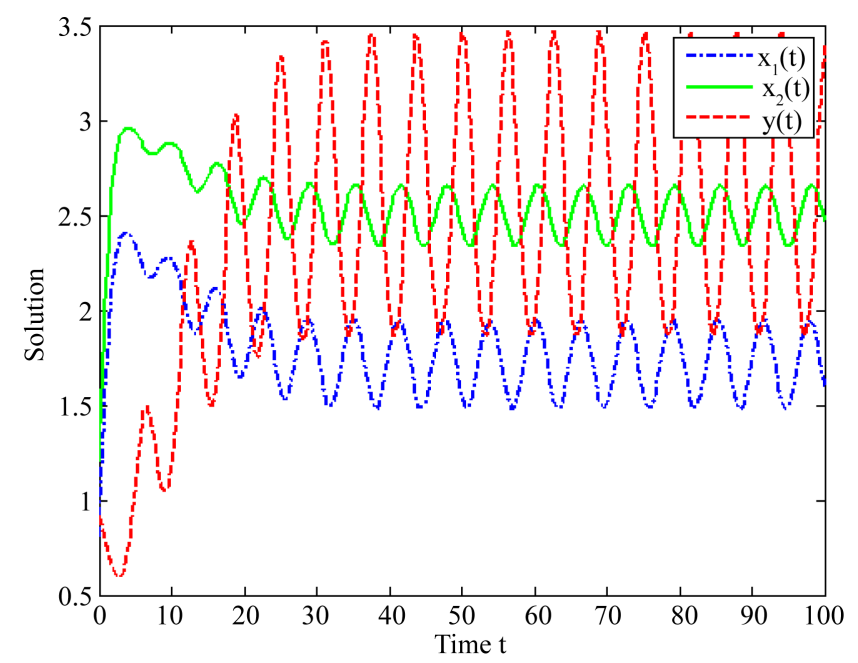

Figure 1. The periodic solution obtained by the numerical integration of system (1.2) with the initial conditions $\left(\phi_{1}(\theta), \phi_{2}(\theta), \psi(\theta)\right)=(1,1,1)$ and $\beta(t)=0.01$, the other parameters given by equation (4.1), where $t \in[0,100]$ and $\theta \in[-0.6,0]$. 


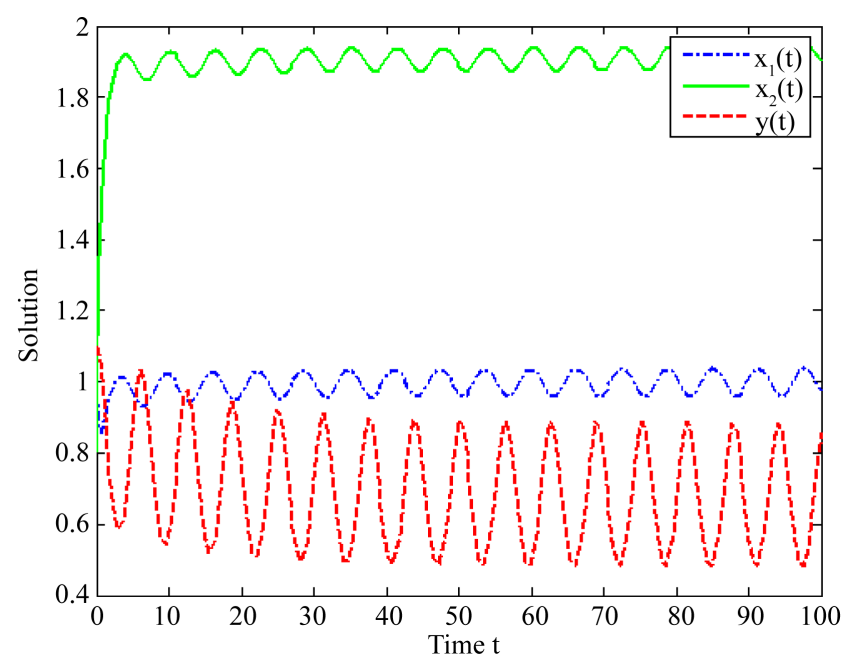

Figure 2. The periodic solution obtained by the numerical integration of system (1.2) with the initial conditions $\left(\phi_{1}(\theta), \phi_{2}(\theta), \psi(\theta)\right)=(1,1,1)$ and $\beta=0.25$, the other parameters given by Equation (4.1), where $t \in[0,100]$ and $\theta \in[-0.6,0]$.

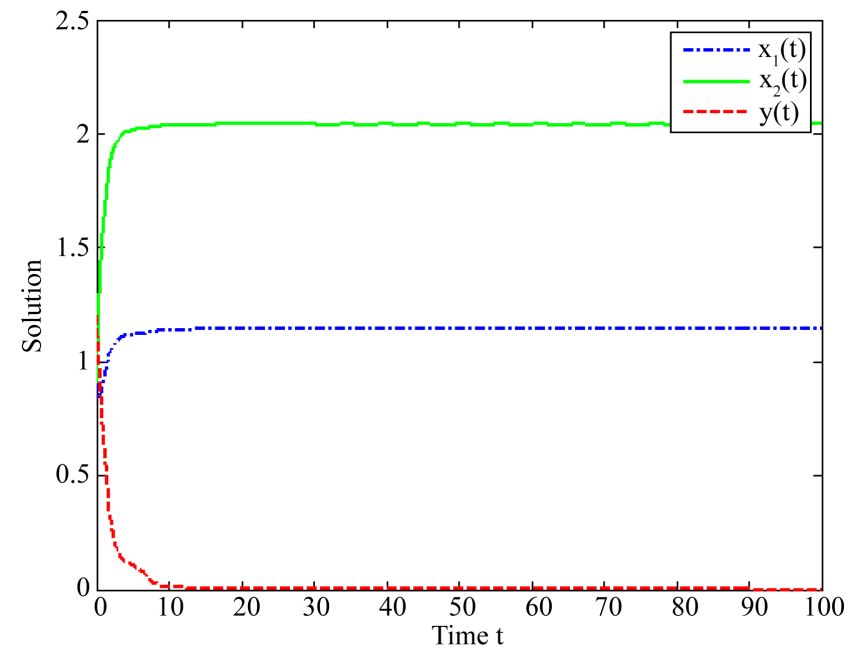

Figure 3. The periodic solution obtained by the numerical integration of system (1.2) with the initial conditions $\left(\phi_{1}(\theta), \phi_{2}(\theta), \psi(\theta)\right)=(1,1,1)$, and $q(t)=0.07$, $d_{3}(t)=0.75+0.3 \sin t$, the other parameters given by Equation (5.1), where $t \in[0,100]$ and $\theta \in[-0.6,0]$.

nile growth. We explore the uniformly persistent and extinction of system (1.2). By Lemma 2.2, we know that $\left(x_{1}^{*}(t), x_{2}^{*}(t)\right)$ is the globally attractive periodic solution of system (1.1) without density dependence and predation. Hence, condition (2.3) implies that system (1.2) is uniformly persistent if the death rate of the predator population is sufficiently small. Numerical simulations not only show the consistency with the theoretical analysis but also exhibit other interesting biological phenomenon. Form Figure 1, Figure 2, we know that although the density dependent coefficient has no influence on the permanence of system (1.2), it affects the system populations' densities.

\section{Funding}

This work was supported by the National Natural Science Foundation of China (No. 31370381), the General 
Project of Educational Commission in Sichuan Province (Grant No. 16ZB0357), the Major Project of Educational Commission in Sichuan Province (Grant No.16ZA0357) and the Major Project of Sichuan University of Arts and Science (Grant No.2014Z005Z).

\section{References}

[1] Liu, S.-Q. and Chen, L.-S. (2002) Recent Progress on Stage-Structured Population Dynamics. Mathematical and Computer Modelling, 36, 1319-1360. http://dx.doi.org/10.1016/S0895-7177(02)00279-0

[2] Xu, R. and Ma, Z.-E. (2008) Stability and Hopf Bifurcation in a Predator-Prey Model with Stage Structure for the Predator. Nonlinear Analysis: Real World Applications, 9, 1444-1460. http://dx.doi.org/10.1016/j.nonrwa.2007.03.015

[3] Liu, C., Zhang, Q.-L. and Huang, J. (2013) Stability Analysis of a Harvested Prey-Predator Model with Stage Structure and Maturation Delay. Mathematical Problems in Engineering, 2013, Article ID: 329592.

[4] Wang, F.-Y. and Pang, G.-P. (2009) The Global Stability of a Delayed Predator-Prey System with Two Stage-Structure. Chaos, Solitons and Fractals, 40, 778-785. http://dx.doi.org/10.1016/j.chaos.2007.08.024

[5] Liu, C., Zhang, Q.-L., Li, J.-N. and Yue, W.-Q. (2014) Stability Analysis in a Delayed Prey-Predator-Resource Model with Harvest Effort and Stage Structure. Applied Mathematics and Computation, 238, 177-192. http://dx.doi.org/10.1016/j.amc.2014.04.015

[6] Meng, X.-Y., Huo, H.-F., Xiang, H. and Yin, Q.-Y. (2014) Stability in a Predator-Prey Model with Crowley-Martin Function and Stage Structure for Prey. Applied Mathematics and Computation, 232, 810-819. http://dx.doi.org/10.1016/j.amc.2014.01.139

[7] Fu, S.-M., Zhang, L.-N. and Hu, P. (2013) Global Behavior of Solutions in a Lotka-Volterra Predator-Prey Model with Prey-Stage Structure. Nonlinear Analysis: Real World Applications, 14, 2027-2045.

http://dx.doi.org/10.1016/j.nonrwa.2013.02.007

[8] Deng, L.-W., Wang, X.-D. and Peng, M. (2014) Hopf Bifurcation Analysis for a Ratio-Dependent Predator-Prey System with Two Delays and Stage Structure for the Predator. Applied Mathematics and Computation, 231, 214-230. http://dx.doi.org/10.1016/j.amc.2014.01.025

[9] Wang, L.-L. and Xu, R. (2013) Global Dynamics of a Predator-Prey Model with Stage Structure and Delayed Predator Response. Discrete Dynamics in Nature and Society, 2013, Article ID: 724325.

[10] Xu, R. and Ma, Z.-E. (2008) Stability and Hopf Bifurcation in a Ratio-Dependent Predator-Prey System with Stage Structure. Chaos, Solitons and Fractals, 38, 669-684. http://dx.doi.org/10.1016/j.chaos.2007.01.019

[11] Shi, R.-Q., Tang, S.-Y. and Feng, W.-L. (2013) Two Generalized Predator-Prey Models for Integrated Pest Management with Stage Structure and Disease in the Prey Population. Abstract and Applied Analysis, 2013, Article ID: 724325.

[12] Fan, X.-L., Teng, Z.-D. and Jiang, H.-J. (2014) Global Property in a Delayed Periodic Predator-Prey Model with Stage-Structure in Prey and Density-Independence in Predator. Abstract and Applied Analysis, 2014, Article ID: 172380.

[13] Wang, X.-H. and Huang, C.-Y. (2014) Permanence of a Stage-Structured Predator-Prey System with Impulsive Stocking Prey and Harvesting Predator. Applied Mathematics and Computation, 235, 32-42. http://dx.doi.org/10.1016/j.amc.2014.02.092

[14] Zhang, L.-M. and Zhang, C.-F. (2010) Rich Dynamic of a Stage-Structured Prey-Predator Model with Cannibalism and Periodic Attacking Rate. Communications in Nonlinear Science and Numerical Simulation, 15, 4029-4040. http://dx.doi.org/10.1016/j.cnsns.2010.02.009

[15] Xu, C.-G. and Chen, D.-X. (2011) Existence and Global Attractivity of Positive Periodic Solutions for a Two-Species Competitive System with Stage Structure and Impulse. International Journal of Differential Equations, 2011, Article ID: 259805.

[16] Huang, C.-Y., Zhao, M. and Zhao, L.-C. (2010) Permanence of Periodic Predator-Prey System with Two Predators and Stage Structure for Prey. Nonlinear Analysis: Real World Applications, 11, 503-514. http://dx.doi.org/10.1016/j.nonrwa.2009.01.001

[17] Li, Z. and Chen, F. (2009) Extinction in Periodic Competitive Stage-Structured Lotka-Volterra Model with the Effects of Toxic. Journal of Computational and Applied Mathematics, 231, 143-153. http://dx.doi.org/10.1016/j.cam.2009.02.004

[18] Yang, W.-S., Li, X.-P. and Bai, Z.-J. (2008) Permanence of Periodic Holling Type-IV Predator-Prey System with Stage Structure for Prey. Mathematical and Computer Modelling, 48, 677-684. http://dx.doi.org/10.1016/j.mcm.2007.11.003 
[19] Abrams, P.-A. and Quince, C. (2005) The Impact of Mortality on Predator Population Size and Stability in Systems with Stage-Structured Prey. Theoretical Population Biology, 68, 253-266. http://dx.doi.org/10.1016/j.tpb.2005.05.004

[20] Chow, Y. and Jang, S.-R.-J. (2012) Cannibalism in Discrete-Time Predator-Prey Systems. Journal of Biological Dynamics, 6, 38-62. http://dx.doi.org/10.1080/17513758.2011.557745

[21] Polis, G.-A. (2003) The Evolution and Dynamics of Intraspecific Predation. Annual Review of Ecology and Systematics, 12, 225-25. http://dx.doi.org/10.1146/annurev.es.12.110181.001301

[22] Zhao, X.-Q. (1991) The Qualitative Analysis of $N$-Species Lotka-Volterra Periodic Competition Systems. Mathematical and Computer Modelling, 15, 3-8. http://dx.doi.org/10.1016/0895-7177(91)90100-L

[23] Cui, J.-A., Chen, L.-S. and Wang, W. (2000) The Effect of Dispersal on Population Growth with Stage-Structure. Computers \& Mathematics with Applications, 39, 91-102. http://dx.doi.org/10.1016/S0898-1221(99)00316-8

[24] Lakshmikantham, V., Matrosov, V.M. and Sivasundaram, S. (1991) Vector Lyapunov Functions and Stability Analysis of Nonlinear Systems. Springer, Dordrecht. http://dx.doi.org/10.1007/978-94-015-7939-1

[25] Zhao, X.Q. (2003) Dynamical Systems in Population Biology. Springer-Verlag, New York. http://dx.doi.org/10.1007/978-0-387-21761-1

[26] Xu, R., Chaplain, M.A.J. and Davidson, F.A. (2005) Permanence and Periodicity of a Delayed Ratio-Dependent Predator-Prey Model with Stage Structure. Journal of Mathematical Analysis and Applications, 303, 602-621. http://dx.doi.org/10.1016/j.jmaa.2004.08.062

[27] Wang, K. and Zhu, Y.-L. (2013) Periodic Solutions, Permanence and Global Attractivity of a Delayed Impulsive Prey-Predator System with Mutual Interference. Nonlinear Analysis: Real World Applications, 14, 1044-1054. http://dx.doi.org/10.1016/j.nonrwa.2012.08.016

\section{Submit or recommend next manuscript to SCIRP and we will provide best service for you:}

Accepting pre-submission inquiries through Email, Facebook, Linkedin, Twitter, etc

A wide selection of journals (inclusive of 9 subjects, more than 200 journals)

Providing a 24-hour high-quality service

User-friendly online submission system

Fair and swift peer-review system

Efficient typesetting and proofreading procedure

Display of the result of downloads and visits, as well as the number of cited articles

Maximum dissemination of your research work

Submit your manuscript at: http://papersubmission.scirp.org/ 